\title{
Helical Assemblies of Gold Nanoparticles
}

\section{G. Daniel Lilly, Ashish Agarwal, Sudhanshu Srivastava, and Nicholas A. Kotov*}

The development of techniques for making helical and semiconductor metallic nano- and microstructures represents the extension of the works of Da Vinci and Archimedes with modern scientific tools. It brings forth interesting application concepts along with tough challenges in optics, biology, and mechanics. Making helical metallic objects with submicrometer pitch lengths comparable to the wavelengths of visible light represents an important technological niche for new optical materials. In this case these objects are expected to have complex 3D topologies and to be small enough to effectively interact with visible light. These features make them difficult to produce with traditional lithographic means. At the same time, they are large enough to exceed the capabilities of the synthetic nanotechnology. For instance, helical assemblies of metal nanoparticles (NPs) could be made by using DNA and similar templates, however they typically have the pitch length in range of few nanometers. ${ }^{[1-5]}$ Other templates based on mostly oxide matrixes can give the helices with typical pitch in the range of tenth of nanometers but not in hundreds of nanometers. ${ }^{[6-8]}$ Lithographic techniques are exceptionally convenient for flat and layered structures but are complicated, expensive, and restrictive for objects with complex 3D topology. The preparation of metal helices with micrometer (and sometimes submicrometer) dimensions is possible, but they are very time-consuming and require unorthodox solutions. ${ }^{[9]}$ A bottom-up approach for making helical metal systems with pitch lengths in $200-900 \mathrm{~nm}$ range would be most desirable from both fundamental and practical points of view.

Besides geometrical elegance, helical structures from plasmonic materials, such as gold or silver, are important research targets because they provide a viable pathway to negative refractive index materials (NIMs). The ability of NIMs to refract light on the opposite side of the normal to the optical interface attracted a lot of attention due to the possibility

Dr. G. D. Lilly, Dr. A. Agarwal, Dr. S. Srivastava, Prof. N. A. Kotov

Department of Chemical Engineering

University of Michigan

Ann Arbor, Michigan 48109, USA

E-mail: kotov@umich.edu

N. A. Kotov

Department of Material Sciences and Engineering

University of Michigan

Ann Arbor MI, 48109, USA

N. A. Kotov

Departments of Biomedical Engineering

University of Michigan

Ann Arbor MI, 48109, USA

DOI: $10.1002 /$ smll.201100536 to construct perfect lenses and cloaking devices. ${ }^{[10-13]}$ The two most actively pursued approaches to NIMs are resonant submicrometer systems and photonic crystals. The first approach typically uses various geometrical arrangements of metallic rods/wires, with or without additional rings, which have overlapping areas of negative permittivity and negative permeability components. ${ }^{[14-16]}$ The size of the resonant submicrometer systems and the metal used for making them determine the wavelengths at which the system would behave as a NIM. By scaling down the size of wire-ring combinations, NIMs could be potentially obtained in the near-IR regime. ${ }^{[17]}$ However, metallic nanowires (NWs) do not behave as metallic conductors in the bulk due to a strong effect of charge-carrier scattering at interfaces and crystallographic grains. ${ }^{[18]}$ This makes further scaling down of the 'light antennas' quite problematic. ${ }^{[9,19]}$ Another major problem with this design is that operating near plasmonic resonances leads to very high optical losses to heating and limits the restoration of evanescent components of light. Compensation of losses by embedding a gain material taking advantage of, for instance off-resonance adsorption of semiconductor nanoparticles (NPs), ${ }^{[20]}$ is possible, but realization of such complex $3 \mathrm{D}$ semiconductor-metal superstructure and the efficiency/coherence of energy transfer in them are hard scientific problems. ${ }^{\text {[21] }}$

The photonic crystals approach to NIMs is based on the excitation of photonic bandgaps with negative slopes to support negative phase velocity in the medium ${ }^{[22]}$ and are limited in their performance due to scattering from surface imperfections. Realization of the all-angle NIM for the visible part of the spectrum based on a photonic crystal is also complicated.

Recent theoretical investigations have showed that NIMs can also be realized through chiral materials under certain conditions. ${ }^{[10,23-26]}$ In a chiral medium, Maxwell's constitutive relations are

$$
\begin{aligned}
& \mathrm{D}=\varepsilon \mathrm{E}+\xi \mathrm{H} \\
& \mathrm{B}=\xi \mathrm{E}+\mu \mathrm{H} \\
& \zeta=\xi *=\mathrm{j} \kappa \operatorname{sqrt}\left(\mu_{0} \varepsilon_{0}\right)
\end{aligned}
$$

where $\mathrm{E}$ is the electrical field strength, B magnetic field strength, D electric flux density (or electric displacement field), $\mathrm{H}$ magnetic flux density (or magnetizing field), $\varepsilon$ electric permittivity, $\mu$ magnetic permeability, $\varepsilon_{0}$ electric permittivity of free space, $\mu_{0}$ magnetic permeability of free space, $\zeta$ and $\xi$ are anisotropy parameters, and $\kappa$ is the dimensionless chirality parameter. Some trivial algebra can show that one of the required conditions to achieve negative refraction is

$|\kappa|>\sqrt{\mu \varepsilon}$ 
A chiral material breaks the degeneracy between two circularly polarized waves, increasing the effective refractive index for one polarization and reducing the refractive index for the other. Zhang et al. demonstrated chiral NIMs using lithographically made metallic chiral resonators $^{[27]}$ and Zheludev et al. demonstrated similar results using twisted planar metal patterns in parallel planes. ${ }^{[28]}$ The size of the resonating structures was on the order of few hundred micrometers and the chiral response was observed around $1 \mathrm{THz}$. The resonating structures in both cases were made by lithographic techniques, thus scaling down becomes challenging.

In this paper a novel method of preparing chiral twisted metallic nanostructures is presented which may find applications for such chiral NIMs, and considerations for the design parameters for such structures are presented. Specifically, twisted CdTe ribbons with microscale length and nanoscale thickness are used. Their helicity originates from the mechanical distortion taking place in flat semiconductor ribbons assembled from NPs. ${ }^{[29]}$ The pitch length is on the submicrometer scale spanning a wide range from 200 to $1000 \mathrm{~nm}$ and can be controlled by the illumination of flat nanowires with
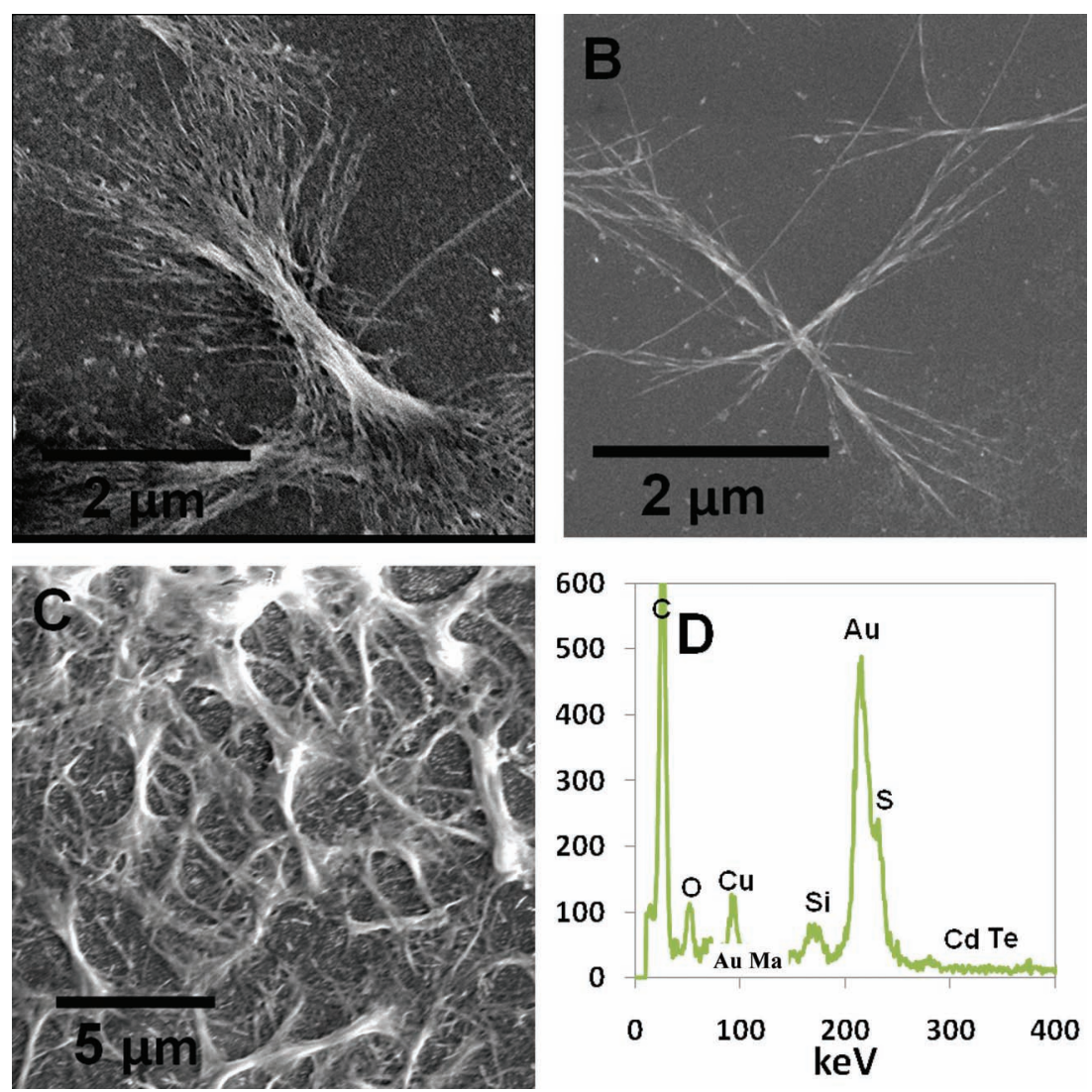

Figure 1. SEM images of A) original twisted CdTe nanoribbons, and their gold replicas obtained after a 2.5 min (B) or 5 min (C) soak in $0.01 \mathrm{M} \mathrm{HAuCl}_{4}$. D) Energy dispersive X-ray (EDS) spectra of Au-plated twisted CdTe nanoribbons shown in (B). light. Twisted ribbons should be applicable

to nanostructures of numerous geometries and compositions, greatly expanding the types of Au nanostructures available to researchers today.

As a starting point for this work we chose CdTe NP assemblies shaped as twisted nanoribbons. ${ }^{[29]}$ They are made from a semiconductor material and therefore cannot be as intense light absorbers and polarizers as metallic nanostructructures. The latter display substantially larger light adsorption cross-sections and faster excitation rates due to the presence of free electron gas electrons and greater polarizabilities. The problem of separation of nanoribbons according to chirality is relegated to a later stage of the project because it is not trivial for nanostructures and will require markedly different approaches than those used for organic materials. Besides knowing whether or not it is possible to prepare the metal helices with submicrometer pitch lengths, we also needed to know their other structural characteristics, which will affect the methods to obtain chiral purity. At this point, the number of nanoribbons with left- and right-twisting was approximately the same: from around 100 twisted nanoribbons, 52 nanoribbons were right handed and 48 nanoribbons had a left-handed twist. ${ }^{[29]}$ In the framework of this study we will be interested only in the transition from semiconductor to metal structures retaining the special 3D topology and in particular the submicrometer pitch.

To convert CdTe NP assemblies to metallic structures, they were treated with a solution of $\mathrm{HAuCl}_{4}$ with the idea that $\mathrm{Te}^{2-}$ would be oxidized, resulting in the deposition of elemental gold onto the original nanoribbons creating a (thin) film. For the purpose of plasmonic resonances in $3 \mathrm{D}$ structures such morphology would have been quite adequate. The results, to some degree exceeded our expectations: the complete replacement of CdTe with $\mathrm{Au}$ (Figure 1 and Figure 2) was observed. The reaction between CdTe NP assemblies and $\mathrm{HAuCl}_{4}$ was rapid and occurred at room temperature. All geometrical features remained unchanged after the CdTe replacement with Au. Particularly of note is that the twisted nature of the nanoribbons, and, therefore, the chirality of individual ribbons are preserved (Figure 1,2). A pitch length of $240 \pm 50 \mathrm{~nm}$ and $220 \pm 30 \mathrm{~nm}$ were observed before and after Au plating, respectively. This instills confidence that the entire range of submicrometer pitch lengths available for semiconductor twisted ribbons, i.e., from 200 to $1500 \mathrm{~nm},{ }^{[29]}$ could also be available for their metal replicas.

As evidenced by the transmission electron microscopy (TEM) data in Figure 2B, the transition from semiconductor to metal helical structure involved initial formation of multiple Au NPs 'sprinkled' over the polycrystalline, twisted CdTe nanoribbons (Figure 2B), which correspond to ca. $30 \mathrm{~s}$ of $\mathrm{HAuCl}_{4}$ treatment. Based on the previous reports regarding $\mathrm{HAuCl}_{4}$ reduction to elemental $\mathrm{Au}$ onto NWs and tetrapods, ${ }^{[32-34]}$ the initial step, i.e., Au nucleation, is likely to occur on the high energy sites of CdTe NPs or at defect sites nanoribbons. Since the twisted nanoribbons used in this 

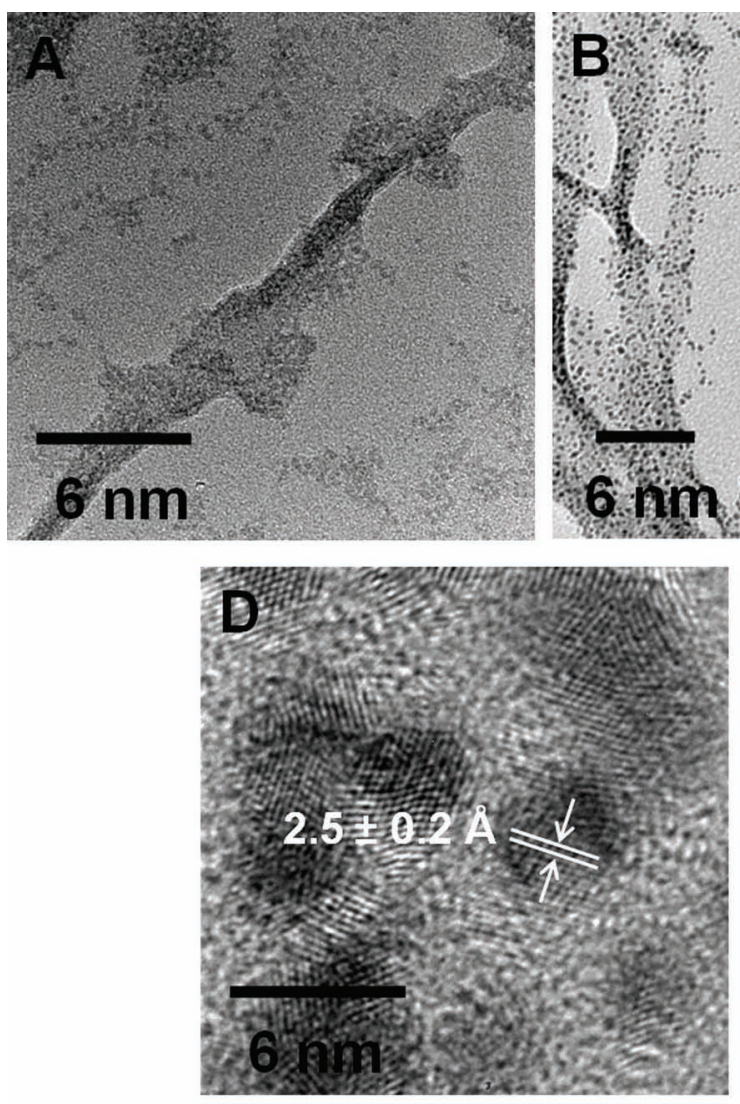
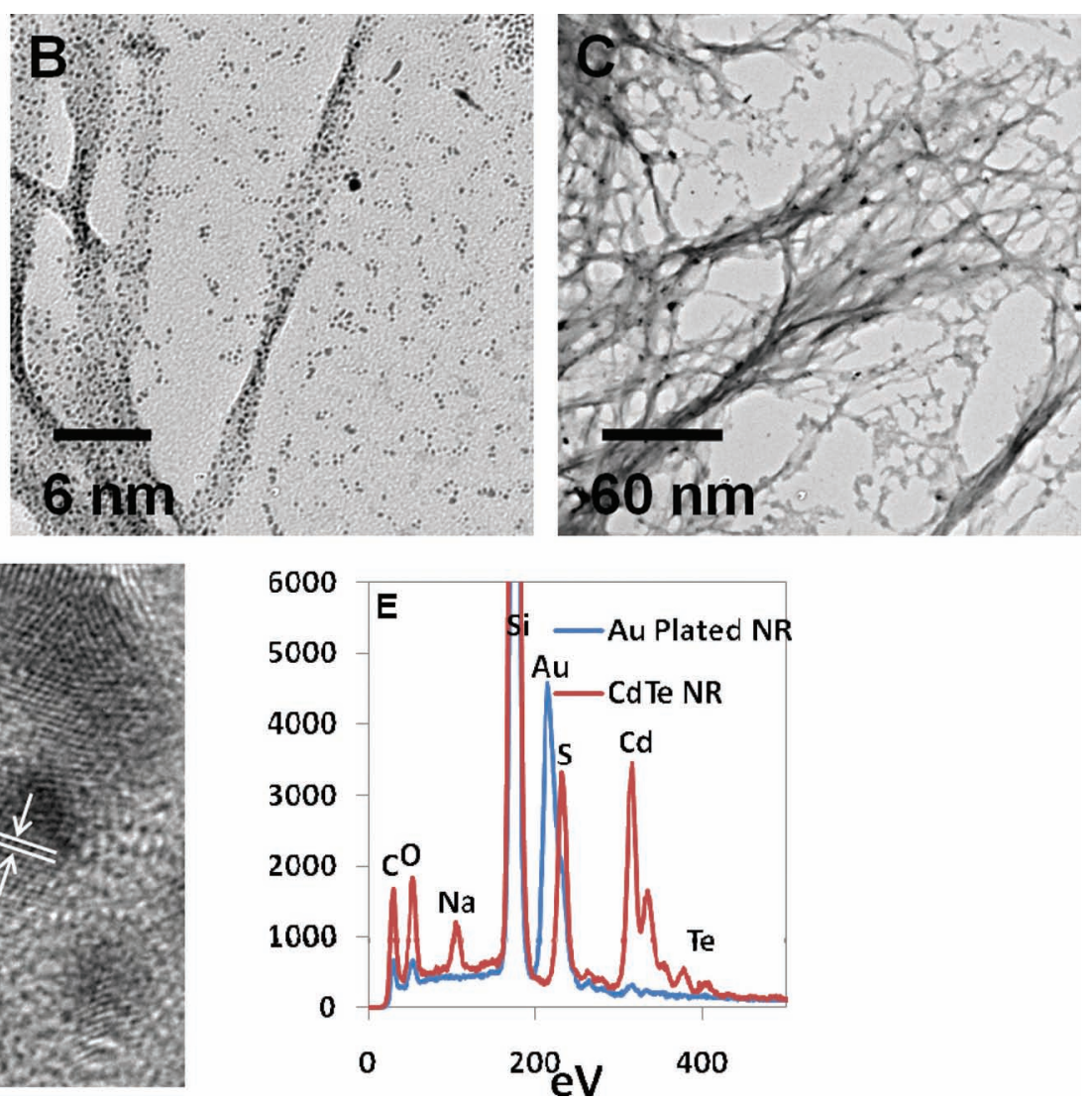

Figure 2. High-resolution (HR)TEM images of A) a twisted (dTe nanoribbon, B) a twisted (dTe nanoribbon soaked in $\left.0.01 \mathrm{~m} \mathrm{HAuCl} \mathrm{for}_{3} 3 \mathrm{~s}, \mathrm{C}\right)$ a twisted CdTe nanoribbon soaked in $0.01 \mathrm{M} \mathrm{HAuCl}_{4}$ for $4 \mathrm{~min}$, D) Au NPs on a twisted CdTe nanoribbon after a $30 \mathrm{~s}$ soak in $0.01 \mathrm{~m} \mathrm{HAuCl} 4$ showing the lattice spacing for Au. E) Elemental mapping of a spotted, twisted nanoribbon showing a the composition of CdTe and spot composition of Au.

work are polycrystalline, ${ }^{[29]}$ there are numerous sites with high interfacial energy to initialize $\mathrm{HAuCl}_{4}$ reduction into atomic $\mathrm{Au}$. The composition of the twisted CdTe nanoribbons and $\mathrm{Au}$ NPs on them was verified by EDS (Figure 2D and E, respectively).

Eventually, these Au NPs merge and form an Au shell surrounding individual nanoribbon (Figure 2C). EDS spectra showed that after 2.5-4.0 min the twisted CdTe NWs were completely converted into $\mathrm{Au}$, with no Au coating on the underlying $\mathrm{Si}$ wafer. No EDS peaks corresponding to $\mathrm{Cd}$ or Te were observed. Further exposure to the $\mathrm{HAuCl}_{4}$ solution results in $\mathrm{Au}$ overplating the twisted nanoribbons which causes the voids between the nanoribbons to fill with $\mathrm{Au}$ (Figure 1C and Figure 2C) and is likely to be related to oxidation of remaining stabilizer molecules in solution.

Considering the versatility of the $\mathrm{HAuCl}_{4}$-based conversion process as well as a wide variety of potential 3D assemblies that CdTe NPs may produce, it is important to develop not only a method of their synthesis but also a potential method of an a priori evaluation of their optical properties as a guide to the synthesis. Computer simulations of optical properties using the CST Microwave Studio were used to evaluate the intensity and spectral range of polarization rotation of the prepared metallic twisted ribbons.

To build up the adequate model one needs to note that like the twisted CdTe nanoribbons themselves, their gold replicas were polycrystalline, which is consistent with the previous studies of the replacement of angled nanostructures from Te with Au. ${ }^{[35]}$ Monocrystalline gold structures might have been expected to be a better choice for NIMs from the stand point of higher electron mobility and stronger plasmon resonance. ${ }^{[18]}$ However, the expectations about the preferred morphology based on intuitive understanding of the phenomenon were actually misleading as demonstrated by the simulations below.

The results from simulations were obtained as a standard scattering matrix, or S-matrix, made of elements corresponding to different scattering modes. In our case of light propagation through the media, the elements in the $S$-matrix are scattering amplitudes determined by the probabilities and cross-sections of different light-matter interactions. One of the elements of this matrix that would be of particular interest in the framework of this study are $S_{Z \max (y), Z \min (y)}$, which describes the transmission of light with electric field polarized along $y$-axis (mode $y$ ) entering at the point of $Z_{\min }$ and exiting at $Z_{\max }$, corresponding to the two ends of the helical structures aligned along $z$-axis. We will also look at the parameters of the $S$-matrix describing to the rotation of the polarization. This is the parameter $S_{\mathrm{Zmax}(x), \mathrm{Zmin}(y)}$, corresponding to mode $y$ entering at $Z_{\min }$ and mode $x$ with electric field polarized along $x$-axis exiting at $Z_{\max }$. Note that $S$-matrix is symmetric and an analogous matrix element $S_{Z \max (y), Z \min (x)}$ 

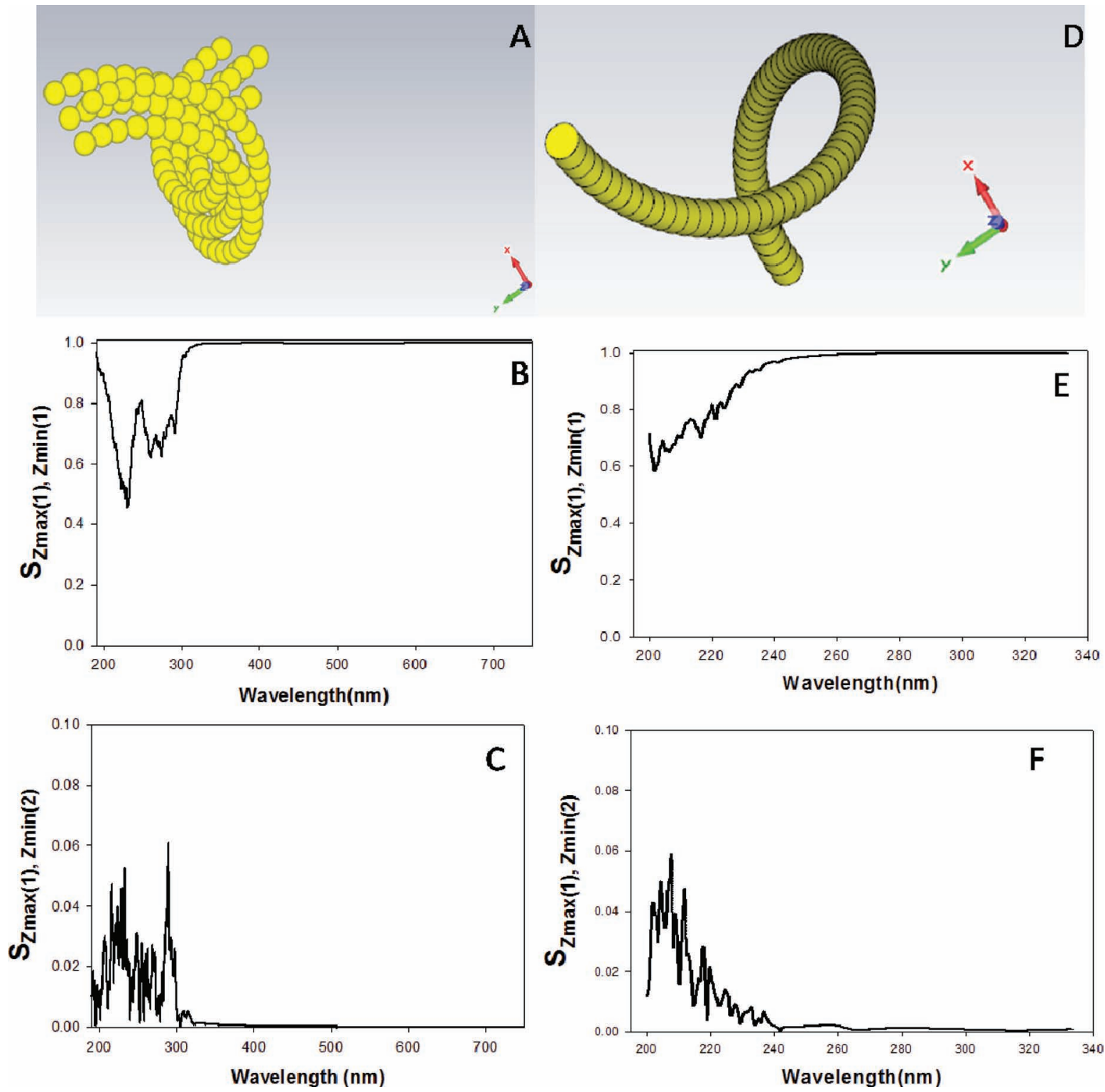

Figure 3. A) Helical assembly of spherical gold NPs as drawn in CST Microwave Studio. B) Wavelength dependence of the matrix element corresponding to transmission of mode $y$ for the structure shown in (A). C) Wavelength dependence of the matrix element corresponding to polarization rotation of mode $x$ into mode $y$ for the structure shown in (A). D) Solid helical gold NW as drawn in CST Microwave Studio. E) Wavelength dependence of the matrix element corresponding to transmission of mode $y$ for the structure shown in (D). F) Wavelength dependence of the matrix element corresponding to polarization rotation of mode $x$ into mode $y$ for the structure shown in (D).

describing mode $x$ entering at $Z_{\min }$ and mode $y$ exiting at $Z_{\max }$ is numerically equal to $S_{Z \max (x), Z \min (y) \text {. }}$

It was observed that for helical assembly of NPs and the solid helical NWs with the same pitch length, the element $\mathrm{S}_{\mathrm{Zmax}(\mathrm{y}), \mathrm{Zmin}(\mathrm{y})}$ describing the transmission of mode $y$ is markedly decreased between $200-320 \mathrm{~nm}$ with the minimum of as low as 0.45 at $230 \mathrm{~nm}$ (Figure 3B). This corresponds closely to the pitch length of the helical assembly prepared, which is $250 \mathrm{~nm}$. As the wavelength of the exciting field moves away from pitch length, the virtually perfect transmission is observed again, i.e., $S_{Z \max (y), Z \min (y)}=1$. This finding is quite important from the perspective of NIMs because it demonstrated that the variation of the pitch length of the helical structures allows one to 'tune' the region of the negative index of refraction, which also coincide the range of pitches possible for CdTe twisted nanoribbons obtained experimentally. ${ }^{[29]}$ Note also the broad range of wavelengths for which decreased transmission was observed, i.e., $200-320 \mathrm{~nm}$. This property needs to be attributed to the discontinuity in the helical structure and the fact that NPs performed as coupled independent oscillators with large light absorption cross-sections typical for NPs. The wider this range, the better it is for NIMs. The region of decreased transmission in helical assemblies of Au NPs is comparable to the spectral range of NIMs made from parallel gold nanorods, i.e., 1100$1400 \mathrm{~nm} \cdot{ }^{[17]}$ In the more conventional terms of quantum optics, this range corresponds to light photons with energy, $v$, from ca. $0.9 \mathrm{eV}$ to $1.1 \mathrm{eV}(\Delta v \approx 0.2 \mathrm{eV})$, while the $200-320 \mathrm{~nm}$ 
range characteristic for the prepared $\mathrm{Au}$ NP helices corresponds to the energy window of 3.9 to $6.2 \mathrm{eV}(\Delta v \approx 2.3 \mathrm{eV})$.

Even considering that the area of the potential NIM activity will be only the central portion of the range of decreased $S_{Z \max (y), Z \min (y)}$, the benefit of having discrete NPs in the helical structure was confirmed by simulations for a solid helical gold NW with the same pitch length (Figure 3D-F). These results also showed much decreased transmission of mode $y$ around the wavelength corresponding to the pitch length of the helices (Figure 3E). However, the spectral range where this polarization-dependent reduction occurred was much narrower, i.e., $200-230 \mathrm{~nm}(\Delta v \approx 0.8 \mathrm{eV})$.

The cross-polarization matrix element, $\mathrm{S}_{\mathrm{Zmax}(\mathrm{y}), \mathrm{Zmin}(\mathrm{x}) \text {, was }}$ used to investigate the cumulative effectiveness of the helical assembly of NPs in rotation of the polarization of light. It was found that for the incoming electric field polarized along the $x$-direction and entering at the point of $Z_{\min }$ and exiting as $y$-polarized light at $Z_{\max }$ of the twisted ribbon from Au NPs, $6 \%$ was rotated and oriented along $y$-direction at the end of the assembly. The spectral region of wavelength where cross-polarization was observed is the same where decreased transmission is observed. Note that overall polarization $6 \%$ produced by a single chirooptical element in space of lightmatter interaction is several times higher than what would be observed from organic materials, such as liquid crystals, in similar conditions. ${ }^{[35]}$

The cross-polarization of the continuous NW was around $5.5 \%$, which is lower than for the case of discrete NPs (Figure 3F). This fact, again, demonstrated the advantage of helical assemblies made from discrete NPs rather than the solid gold continuous structures.

In conclusion, we have demonstrated that helical metallic structures with submicrometer pitch length can be produced using fast, simple, and versatile process of $\mathrm{HAuCl}_{4}$ treatment. Taking advantage of a variety of CdTe templates and a range of available pitch lengths it can potentially lead to tunable NIMs and other chiro-optical materials. Looking ahead at the future effort on realization of chiral NIMs in visible range of light spectrum, the helical assemblies of individual NPs 1) appear to be a viable research undertaking and 2) would potentially be preferred to helical nanowires from solid gold due to wider range of chiro-optical response as demonstrated by simulations provided. Major effort now should be directed toward realization of chiral purity of the prepared submicrometer helices either from semiconductors, metals or even oxides which will require innovative synthetic or separation approaches. Since Au NPs can be easily deposited on many templates, such morphology would be quite adequate for NIMs or other materials utilizing strong polarization rotation.

\section{Experimental Section}

Chemicals: $\mathrm{HAuCl}_{4}$ (gold(III) chloride hydrate), $\mathrm{Cd}\left(\mathrm{ClO}_{4}\right)_{2} \cdot \mathrm{H}_{2} \mathrm{O}$ (cadmium perchlorate), $\mathrm{CdO}$ (cadmium oxide), $\mathrm{C}_{2} \mathrm{H}_{4} \mathrm{O}_{2} \mathrm{~S}$ (thioglycolic acid, TGA), $\mathrm{CH}_{3} \mathrm{OH}$ (methanol), $\left(\mathrm{CH}_{3}\right)_{2} \mathrm{CHOH}$ (2-propanol), and $\left(\mathrm{C}_{8} \mathrm{H}_{16} \mathrm{CIN}\right)_{n}$ (poly(diallyldimethylammonium chloride), PDDA), $\mathrm{H}_{2} \mathrm{O}_{2}$ (hydrogen peroxide), and $\mathrm{H}_{2} \mathrm{SO}_{4}$ (sulfuric acid) were obtained from
Sigma-Aldrich and used without further purification; $\mathrm{Al}_{2} \mathrm{Te}$ (aluminum telluride) was obtained from Cerac, Inc and used without further purification; $\mathrm{NaOH}$ (sodium hydroxide) was obtained from Fluka and used without further purification; and all water was purified using $18 \mathrm{M} \Omega$ deionized water (Barnstead E-pure system).

Synthesis and Characterization of the Gold Helices: Twisted $\mathrm{CdTe}$ nanoribbons were produced according to a procedure in the literature. ${ }^{[29]}$ The twisted NWs were produced from CdTe NPs with a reduced ratio of TGA stabilizer for the CdTe NPs in the initial synthetic step. The assembly process was allowed to proceed under ambient light conditions, since it is known that light can cause defects such as truncations on the surface of the NPs that can change the magnitude and direction of the NP charge dipoles leading to interesting assemblies. CdTe NP dispersions were prepared using the procedure from Gaponik et al. ${ }^{[30]}$ with the fraction of TGA to $\mathrm{Cd}^{2+}$ close to 1.0 in ratio. $1.095 \mathrm{~g}$ of $\mathrm{Cd}\left(\mathrm{ClO}_{4}\right)_{2} \cdot 6 \mathrm{H}_{2} \mathrm{O}$ was dissolved in $200 \mathrm{~mL}$ of pure water. TGA was added into the solution at a molar ratio of $\sim 1.0$ against $\mathrm{Cd} 2+$. The $\mathrm{pH}$ values of the solution were adjusted to 11.4 by the drop-wise addition of $1 \mathrm{~m} \mathrm{NaOH}$ solution. The solution was deoxygenated by bubbling $\mathrm{N}_{2}$ through the solution for $60 \mathrm{~min}$. Under stirring, $\mathrm{H}_{2} \mathrm{Te}$ gas was passed through the solution with a slow nitrogen flow for $60 \mathrm{~min}$. The precursors were converted to CdTe NPs by refluxing at approximately $100{ }^{\circ} \mathrm{C}$ for $50 \mathrm{~min}$. Dispersions with a luminescence peak of $550 \mathrm{~nm}$ were obtained. The CdTe NPs were then precipitated by addition of methanol and centrifuged for $20 \mathrm{~min}$. The CdTe NPs were redispersed in pure water at $\mathrm{pH} 9$ (modified by the addition of $\mathrm{NaOH}$ ). The dispersion was exposed to ambient light and aged. The orange solution of NPs turned dark green in about $48 \mathrm{~h}$, which differs from our previous studies where the dispersions turned dark brown. Upon analysis, the samples showed distinctive helical structures and straight NWs. The twisted CdTe nanoribbons were then immobilized using layer-by-layer deposition. ${ }^{[31]}$ For scanning electron microscopy (SEM) characterization, piranha-cleaned $\mathrm{Si}$ wafers (wafers soaked in a mixture of $30 \%(\mathrm{v} / \mathrm{v}) \mathrm{H}_{2} \mathrm{O}_{2}$ and $70 \%$ ( $(\mathrm{v} / \mathrm{v}) \mathrm{H}_{2} \mathrm{SO}_{4}$ for $30 \mathrm{~min}$ and rinsed) are used as substrates; for TEM characterization, carbon on carbon TEM grids supplied by Ted Pella, Inc. are used as substrates. The substrate is then soaked in $0.5 \%(\mathrm{v} / \mathrm{v})$ PDDA for $5 \mathrm{~min}$, rinsed in water, and air dried to create a thin, positively charged film on the substrate to which the negatively charged twisted nanoribbons can adhere. The substrate is then soaked in the twisted nanoribbon solution for $1 \mathrm{~min}$, rinsed in water and air dried. The resulting substrates have a good dispersion of twisted CdTe nanoribbons (Figure 1 and Figure 2). Once the twisted $\mathrm{CdTe}$ nanoribbons are immobilized, they are plated in $\mathrm{Au}$ by soaking the substrate in $0.01 \mathrm{~m} \mathrm{HAuCl} 4$ for between $30 \mathrm{~s}$ and $5 \mathrm{~min}$, at which time it is rinsed in water and air dried.

SEM was conducted with a FEI Nova Nanolab Dualbeam Focused Ion Beam Workstation and scanning electron microscope and a Philips XL30FEG. TEM and scanning transmission electron microscopy (STEM) were conducted with a JEOL 2010F high-resolution electron microscope. Energy dispersive X-ray microanalysis (EDS) was conducted with EDAX XEDS detectors on both the Philips XL30FEG SEM and the JEOL 2010F TEM. Centrifugation was performed with a Fisher Scientific Marathon $26 \mathrm{~K} \mathrm{M}$ centrifuge with a Hermile 220.97.V02 rotor.

Computer Simulations: CST Microwave Studio (version 2010) was used to simulate the electric field response of the helical assembly of gold nanoparticles. The helical structure was drawn by 
placing $5 \mathrm{~nm}$-diameter spherical gold nanoparticles separated by $2 \mathrm{~nm}$ along the path of a helix with a pitch of $250 \mathrm{~nm}$ and radius $15 \mathrm{~nm}$. The material properties of each gold NP were described by the Drude model. The surrounding material was assumed to be vacuum with the boundary conditions being a unit cell in the $x, y$-directions and open in $z$-direction. Tetrahedral meshing with 30 steps per wavelength yielding 205035 tetrahedrons cells was used. The length of the wire is along the $z$-axis and the boundary conditions are placed at $Z_{\min }$ and $Z_{\max }$ corresponding to the two ends of the wire. Frequency domain solver, with two Transverse Electric (TE) modes allowed on each $Z_{\min }$ and $Z_{\max }$ point (also denoted in the software as 'ports'), was used to solve the geometry. The excitation signal was at $Z_{\min }$ with mode $y$ having an electric field polarized along the $y$-axis and mode $x$ having an electric field polarized along $x$-axis. To make the comparison in electric field responses a solid wire was also studied. The wire was drawn by extending a circle along a linear spiral curve with pitch length of $250 \mathrm{~nm}$, coil radius $15 \mathrm{~nm}$, and segmentation angle $5^{\circ}$. The material properties of the wire are described by the Drude model. The surrounding material is assumed as vacuum with the boundary conditions being unit cell in the $x, y$-directions and open in the $z$-direction. Tetrahedral meshing with 30 steps per wavelength yielding 95000 tetrahedral cells was used. The boundary conditions, port modes, and excitation source were the same as in the previous case.

\section{Acknowledgements}

The primary support for this work is by AFOSR MURI 444286P061716. The partial support by the Center for Solar and Thermal Energy Conversion, an Energy Frontier Research Center funded by the U.S. Department of Energy, Office of Science, Office of Basic Energy Sciences under Award Number \#DE-SC0000957 as well as NSF grants ECS-0601345; EFRI-BSBA 0938019; CBET 0933384; and CBET 0932823 is also acknowledged. Any opinions, findings, and conclusions or recommendations expressed in this material are those of the authors and do not necessarily reflect the views of the National Science Foundation (NSF). The authors thank the University of Michigan's EMAL for its assistance with electron microscopy, and for the NSF grant \#DMR-9871177 for funding for the JEOL 2010F analytical electron microscope used in this work.

This Communication is part of the Special Issue dedicated to Chad Mirkin in celebration of 20 years of influential research at Northwestern University.

[1] M. Yang, N. A. Kotov, J. Mater. Chem. 2011, 21, 6775.

[2] J. Fei, Y. Cui, A. Wang, P. Zhu, J. Li, Chem. Commun. 2010, 46, 2310.
[3] M. Kimura, T. Hatanaka, H. Nomoto, J. Takizawa, T. Fukawa, Y. Tatewaki, H. Shirai, Chem. Mater. 2010, 22, 5732.

[4] M. J. Bierman, Y. K. A. Lau, A. V. Kvit, A. L. Schmitt, S. Jin, Science 2008, 320, 1060.

[5] X. Su, R. Kanjanawarut, ACS Nano 2009, 3, 2751.

[6] H. Ogihara, M. Sadakane, W. Ueda, Topics in Applied Physics: Inorganic and Metallic Nanotubular Materials 2010, 117, 147.

[7] C. Sanchez, E. L. Crepaldi, A. Bouchara, F. Cagnol, D. Grosso, G. J. D. A. A. Soler-Illia, Mater. Res. Soc. Symp. Proc. 2002, 728, 3.

[8] F. H. Schacher, T. Rudolph, M. Drechsler, A. H. E. Mueller, Nanoscale 2011, 3, 288.

[9] B. A. Korgel, Science 2005, 309, 1683.

[10] a) J. B. Pendry, Science 2004, 306, 1353; b) R. Liu, C. Ji, J. J. Mock, J. Y. Chin, T. J. Cui, D. R. Smith, Science 2009, 323, 366.

[11] J. B. Pendry, Phys. Rev. Lett. 2000, 85, 3966.

[12] S. A. Cummer, B.-I. Popa, D. Schurig, D. R. Smith, J. Pendry, Phys. Rev. E 2006, 74, 036621/1.

[13] I. I. Smolyaninov, Y.-J. Hung, C. C. Davis, Science 2007, 315, 1699.

[14] R. A. Shelby, D. R. Smith, S. Schultz, Science 2001, 292, 77.

[15] T. J. Yen, W. J. Padilla, N. Fang, D. C. Vier, D. R. Smith, J. B. Pendry, D. N. Basov, X. Zhang, Science 2004, 303, 1494.

[16] S. Linden, C. Enkrich, M. Wegener, J. Zhou, T. Koschny, C. M. Soukoulis, Science 2004, 306, 1351.

[17] V. M. Shalaev, W. Cai, U. K. Chettiar, H.-K. Yuan, A. K. Sarychev, V. P. Drachev, A. V. Kildishev, Opt. Lett. 2005, 30, 3356.

[18] K. Critchley, B. P. Khanal, M. L. Gorzny, L. Vigderman, S. D. Evans, E. R. Zubarev, N. A. Kotov, Adv. Mater. 2010, 22, 2338.

[19] C. M. Soukoulis, Science 2007, 315, 1077.

[20] A. Agarwal, G. D. Lilly, A. O. Govorov, N. A. Kotov, J. Phys. Chem. C 2008, 112, 18314.

[21] V. M. Shalaev, Nat. Photonics 2007, 1, 41.

[22] T. Taubner, D. Korobkin, Y. Urzhumov, G. Shvets, R. Hillenbrand, Science 2006, 313, 1595.

[23] Q. Cheng, T. J. Cui, Phys. Rev. B 2006, 73, 113104/1.

[24] T. G. Mackay, Microw. Opt. Techn. Lett. 2005, 45, 120.

[25] J. Q. Shen, S. L. He, J. Phys. A 2006, 39, 457.

[26] Y. Jin, S. L. He, Opt. Express 2005, 13, 4974.

[27] S. Zhang, Y.-S. Park, J. Li, X. Lu, W. Zhang, X. Zhang, Phys. Rev. Lett. 2009, 102, 023901/1.

[28] E. Plum, J. Zhou, J. Dong, V. A. Fedotov, T. Koschny, C. M. Soukoulis, N. I. Zheludev, Phys. Rev. B 2009, 79, 035407/1.

[29] S. Srivastava, A. Santos, K. Critchley, K. S. Kim, P. Podsiadlo, K. Sun, J. Lee, C. L. Xu, G. D. Lilly, S. C. Glotzer, N. A. Kotov, Science 2010, 327, 1355.

[30] N. Gaponik, D. V. Talapin, A. L. Rogach, K. Hoppe, E. V. Shevchenko, A. Kornowski, A. Eychmueller, H. Weller, J. Phys. Chem. B 2002, 106, 7177.

[31] A. L. Rogach, N. A. Kotov, D. S. Koktysh, A. S. Susha, F. Caruso, Coll. Surf. A Phys. Eng. Aspects 2002, 202, 135.

[32] D. V. Talapin, H. Yu, E. V. Shevchenko, A. Lobo, C. B. Murray, J. Phys. Chem. C 2007, 111, 14049.

[33] T. Mokari, E. Rothenberg, I. Popov, R. Costi, U. Banin, Science 2004, 304, 1787.

[34] A. E. Saunders, I. Popov, U. Banin, J. Phys. Chem. B 2006, 110, 25421.

[35] S. Furumi, Chem. Rec. 2010, 10, 394.

Received: March 19, 2011

Published online: June 22, 2011 